\title{
Métodos indiretos de estimação da cobertura de dossel em povoamentos de clone de eucalipto
}

\author{
Marco Antonio Monte ${ }^{(1)}$, Maria das Graças Ferreira Reis ${ }^{(1)}$, Geraldo Gonçalves dos Reis ${ }^{(1)}$, \\ Hélio Garcia Leite ${ }^{(1)}$ e Jonathan James Stocks ${ }^{(1)}$
} (1)Universidade Federal de Viçosa, Dep. de Engenharia Florestal, CEP 36570-000 Viçosa, MG. E-mail: marcomonte@vicosa.ufv.br,
mgfreis@ufv.br, greis@ufv.br, hgleite@ufv.br, jjstocks_br@yahoo.com.br

\begin{abstract}
Resumo - O objetivo deste trabalho foi avaliar o potencial de uso de fotografias digitais na estimação do índice de cobertura de dossel (ICD), em substituição ao índice de área foliar (IAF). Fotografias digitais de 640x480 e de 1.280x960 pixels foram processadas por meio dos aplicativos Erdas, Gap Light Analyzer e Sidelook. O IAF, estimado com o LAI-2000, e as fotografias digitais foram obtidos aos 81 e 93 meses de idade, em povoamento de clone de eucalipto submetido à desrama e ao desbaste. Foram observadas correlações significativas entre IAF e ICD, e as fotografias com maiores dimensões permitiram maior diferenciação entre céu e dossel. O ICD, obtido pela classificação das fotografias com o aplicativo Sidelook, apresentou correlação mais elevada com o IAF, em relação ao obtido com o Erdas e o Gap Light Analyzer. Esses resultados indicam que fotografias digitais podem ser utilizadas para estimar o ICD de povoamentos florestais.
\end{abstract}

Termos para indexação: Eucalyptus grandis, índice de área foliar, fotografia digital, analisador de dossel, estrutura de copa.

\section{Indirect methods to estimate canopy coverage of clonal eucalypt plantations}

\begin{abstract}
The objective of this work was to evaluate methods to process digital photographs in order to estimate the canopy coverage index (CCI), to replace the use of leaf area index (LAI). Digital photographs with 640x480 and 1,280x960 pixels were processed with the Erdas Imagine, Gap Light Analyzer and Sidelook softwares. Leaf area index, using the LAI-2000, and digital photographs were obtained from pruned and thinned Eucalyptus grandis clone plantations at 81 and 93 months of age. Significant correlations were observed between LAI and CCI. Greater sized photographs (1,280x980 pixels) allowed better differentiation between sky and canopy pixels. Canopy Coverage Index obtained from digital photographs processed with the Sidelook was better correlated with LAI than that obtained by Erdas Imagine and Gap Light Analyzer. It is possible to use digital photography for eucalypt forest CCI estimation.
\end{abstract}

Index terms: Eucalyptus grandis, leaf area index, digital photography, plant canopy analyser, crown structure.

\section{Introdução}

A desrama artificial e o desbaste influenciam a arquitetura de copa das árvores e a estrutura do dossel, com reflexo nas condições microclimáticas no interior de uma floresta, especialmente por regularem a quantidade de luz que atinge os estratos médio e inferior do dossel (Almeida, 2003; Chaves, 2005).

A quantidade da radiação interceptada pela floresta é determinada por características da copa, assim como pela localização e pelo tamanho das clareiras existentes no dossel (Hardy et al., 2004). O índice de área foliar (IAF) é um parâmetro importante na avaliação da estrutura do dossel, por caracterizar sua arquitetura e estar relacionado com a produção de biomassa vegetal (Linhares et al., 2000; Villa Nova et al., 2003; Xavier \& Vettorazzi, 2003).

A estimativa do IAF é essencial para determinar e modelar as características ambientais do dossel (Macfarlane et al., 2000; Leblanc \& Chen, 2001). Sua estimativa pode ser alcançada por métodos diretos e métodos indiretos (Cherry et al., 1998; Macfarlane et al., 2000; Jonckheere et al., 2004; Weiss et al., 2004).

O método direto, necessário para a calibração dos demais, é trabalhoso, pois necessita da coleta de material foliar para determinação da área foliar (Cherry et al., 1998). 
Os métodos indiretos (não-destrutivos) são bastante utilizados por serem mais práticos. Dos equipamentos existentes para obtenção indireta do IAF, destaca-se o analisador de dossel LAI-2000 da LI-COR, que utiliza lentes hemisféricas (Linhares et al., 2000; Xavier et al., 2002; Almeida, 2003; Lima, 2003; Xavier \& Vettorazzi, 2003; Chaves, 2005; Pezzopane et al., 2005), por sua praticidade e rapidez na obtenção dos dados, além de fornecer estimativa confiável do IAF, apesar do seu elevado custo. O princípio de uso desse aparelho consiste no balanço entre a radiação que atinge o interior do dossel e a radiação que chega ao topo do dossel, o que é determinado pelo grau de cobertura do dossel, que por sua vez é associado à densidade foliar e, conseqüentemente, ao IAF. A obtenção de leituras ocorre sob radiação difusa, evitando-se a coleta de dados sob radiação direta.

Fotografias hemisféricas ou digitais comuns constituem uma alternativa para o cálculo do IAF, do balanço de radiação e da arquitetura do dossel (Macfarlane et al., 2000; Jonckheere et al., 2004; Chaves, 2005; Leblanc et al., 2005; Zhang et al., 2005; Przeszlowska et al., 2006). Fotografias digitais comuns foram utilizadas por Chaves (2005) e Przeszlowska et al. (2006), respectivamente, para obtenção do índice de cobertura de dossel (ICD), em povoamentos de eucalipto, e do índice de área verde em pastagem. Esses autores utilizaram o aplicativo Erdas na classificação das fotografias e recomendaram que melhorias fossem realizadas para refinamento dessa técnica, uma vez que constitui uma alternativa mais econômica.

Zhang et al. (2005) propuseram a utilização de exposição que permitisse melhor diferenciação entre céu e dossel, enquanto Nobis \& Hunziker (2005) sugeriram melhorar o método de classificação dos pixels que representam o céu e o dossel durante o processamento da fotografia. Nesse caso, o passo inicial seria a transformação binária, adotando-se limiarização manual (Wagner, 1998, 2001). Contudo, a escolha manual do limiar é subjetiva e arbitrária, e representa uma fonte de erro (Frazer et al., 2001; Jonckheere et al., 2004).

Nobis \& Hunziker (2005) sugeriram um algoritmo para determinação automática do limiar, baseado na detecção do valor da borda, dado pelo mais alto contraste entre os pixels que representam o céu e o dossel, e concluíram que este algoritmo é apropriado para substituir o método manual, por requerer menor tempo na análise, o que permite que seja aplicado em quantidade maior de fotografias, com maior precisão. Este algoritmo foi implementado no aplicativo Sidelook 1.1 (Nobis, 2005).

Este trabalho teve como objetivo avaliar o potencial de uso de fotografias digitais para estimar o índice de cobertura de dossel, em substituição ao índice de área foliar.

\section{Material e Métodos}

Os dados foram obtidos em um povoamento de Eucalyptus grandis W. Hill ex Maiden (clone 24504) estabelecido em novembro de 1998, no espaçamento inicial de 3x3 m, no Município de Abaeté, MG, região de Cerrado, situado à latitude $19^{\circ} 15^{\prime} 94^{\prime \prime} \mathrm{S}$ e à longitude $45^{\circ} 44^{\prime} 56^{\prime \prime} \mathrm{O}$, em um Latossolo Vermelho-Amarelo. A temperatura média anual é de $20,7^{\circ} \mathrm{C}$; a precipitação média anual, de $1.350 \mathrm{~mm}$, com valor médio de deficit hídrico anual de $142 \mathrm{~mm}$, entre abril e outubro; e a evapotranspiração potencial anual, de $1.216 \mathrm{~mm}$, relativa ao período de 1999 a 2005.

As plantas desse povoamento foram submetidas à desrama artificial até a altura de 6 m entre 16 e 45 meses (Lima, 2003). Aos 55 meses de idade, foi realizado um desbaste seletivo, eliminando-se 35\% do total de mudas plantadas, o que correspondeu a, aproximadamente, $26,9 \%$ da área basal. A distribuição espacial das plantas eliminadas foi analisada a fim de evitar a formação de grandes clareiras (Chaves, 2005). Foram utilizadas 18 parcelas constituídas de quatro linhas cada, com 18 plantas na linha. Os dados de IAF e a tomada das fotografias foram obtidos em dois pontos de amostragem por parcela, localizados no ponto de interseção de quatro plantas das duas linhas centrais (entre a $6^{\mathrm{a}} \mathrm{e}$ 7 $\mathrm{a}$ e entre a $12^{\text {a }}$ e $13^{\text {a }}$ árvore, a partir do início da parcela), o que totalizou 36 pontos de amostragem.

O IAF foi estimado, aos 81 e 93 meses de idade, com o analisador de dossel da LI-COR (LAI-2000), utilizando um sensor LI-2050 instalado em área aberta próximo às parcelas, e, outro, usado para obtenção dos dados no interior do povoamento. As leituras foram realizadas somente quando havia luz difusa, entre 6 e $7 \mathrm{~h}$ e entre 17 e $17 \mathrm{~h} 40$. As leituras foram obtidas sem o uso de máscaras, uma vez que testes preliminares indicaram não haver efeito significativo de borda. Segundo Lima (2003), aos 45 meses de idade não havia diferença significativa no IAF desse povoamento entre os tratamentos de desrama, em razão da rápida recuperação da copa do clone estudado. 
O ICD foi obtido a partir da tomada de fotografias digitais coloridas a $0,5 \mathrm{~m}$ do solo nos mesmos pontos de amostragem do IAF. A câmera foi colocada sobre um tripé de madeira e orientada na direção da entrelinha, com a lente apontada para o céu. Aos 81 meses de idade, as fotografias foram obtidas com uma câmera Sony MVC FD88, com dimensões de 640x480 pixels, ao passo que, aos 93 meses, foi utilizada uma câmera Sony W7 e dimensões de $1.280 \times 960$ pixels, sempre no modo automático. As fotografias foram registradas na parte da manhã, entre 7 e $9 \mathrm{~h} 30$ e, na parte da tarde, entre 16 e 17h30, evitando-se horários de elevada incidência de radiação solar e, também, a influência de ventos fortes que movimentam a copa das árvores.

As fotografias digitais foram classificadas por meio dos aplicativos Erdas Imagine 8.5 (Leica Geosystems, 1999), Gap Light Analyzer-GLA 2.0 (Frazer et al., 1999) e Sidelook 1.1 (Nobis, 2005) para obtenção do ICD. Selecionou-se o aplicativo que permitiu melhor classificação da fotografia - separação entre pixels que representam o céu e o dossel - por meio da correlação entre ICD e IAF.

O processamento das fotografias digitais com o aplicativo Erdas envolveu a classificação automática nãosupervisionada por meio do algoritmo isodata, considerando-se inicialmente quatro classes de níveis de cinza. A tonalidade mais clara representava a porção de céu visível, enquanto as mais escuras representavam elementos do dossel, como galhos, folhas e troncos, que foram agrupados para representar a cobertura de dossel. Maior número de classes de níveis de cinza facilita a separação entre céu e dossel, principalmente pelo fato de as folhas apresentarem diferentes graus de transmissão da radiação e poderem ser classificadas em diferentes tonalidades. Foi utilizada a convergência de limiar do próprio aplicativo $(0,95)$, com o máximo de seis iterações. Em algumas fotografias, que apresentavam grande proporção de nuvens, foram adotadas seis classes de níveis de cinza, a fim de obter uma estimativa mais precisa do ICD, e evitar que nuvens fossem classificadas como cobertura vegetal.

Na classificação das fotografias com o GLA 2.0, foi utilizada somente a banda azul das fotografias, por apresentar maior contraste entre céu e dossel, com base em testes preliminares com as demais fotografias deste estudo. A determinação do limiar foi realizada manualmente e definida anteriormente por meio da classificação de algumas fotografias, com comparação visual da fotografia original com a classificada na banda azul. Assim, definiu-se um limiar comum, que foi de 145 para fotografias obtidas aos 81 meses, e de 160 para fotografias obtidas aos 93 meses. Por meio desse aplicativo, é obtida uma imagem binária, preto-e-branco, das fotografias.

O aplicativo Sidelook 1.1 permite a classificação das fotografias nas bandas azul, vermelho ou verde e oferece, ainda, a alternativa de fazer essa classificação em níveis de cinza. A banda azul e níveis de cinza foram utilizadas neste trabalho, com base em testes preliminares, por permitir melhor diferenciação entre céu e dossel. Após a classificação, foram obtidas imagens binárias.

As diferentes formas de classificação das fotografias, utilizando esse aplicativo, são descritas a seguir: níveis de cinza e determinação manual do limiar, com uso do ponto máximo do valor de borda (SDL1); níveis de cinza e determinação automática do limiar, levando-se em conta o ponto máximo do valor de borda, com uso da função "next" (SDL2); banda azul e determinação manual do limiar, sem considerar o valor de borda (SDL3); banda azul e determinação automática do limiar (SDL4); banda azul e determinação automática do limiar, levando-se em conta o ponto máximo do valor de borda, com uso da função "next" (SDL5).

Nos dois últimos métodos, foi selecionada outra banda para fotografias que apresentavam distorção decorrente da presença de nuvens ou excesso de luminosidade.

Em todos os casos, os ICD foram obtidos pela razão entre a quantidade de pixels que representava a cobertura vegetal e o total de pixels da fotografia.

Foi estimado o coeficiente de correlação de Pearson, a 5\% de probabilidade, para comparar o IAF com os valores de ICD. Também foram ajustadas equações de regressão a fim de estimar o IAF com o ICD proveniente de cada método de classificação das fotografias digitais.

\section{Resultados e Discussão}

Aos 81 meses de idade, houve correlação significativa entre os valores de ICD e de IAF quando as fotografias foram classificadas utilizando os vários métodos com o aplicativo Sidelook 1.1 (Tabela 1). A correlação foi maior ao se classificar a fotografia utilizando níveis de cinza (SDL1 e SDL2) em comparação com a banda azul.

Nobis \& Hunziker (2005), visando a determinar o algoritmo para determinação automática do limiar no Sidelook 1.1, adotaram a banda azul na análise de fotografias hemisféricas, por considerá-la a mais eficiente 
na separação entre céu e dossel. Neste trabalho, para os dados obtidos aos 81 meses de idade, esta banda foi testada para obtenção do ICD (SDL3, SDL4 e SDL5), mas não apresentou resultados tão satisfatórios, quando comparados com os obtidos com os níveis de cinza (SDL1 e SDL2).

Ao se comparar os ICD obtidos por meio de diferentes métodos com o aplicativo Sidelook 1.1, observa-se elevada correlação entre eles (Tabela 1); as correlações mais baixas foram observadas em relação ao SDL4, que não apresentou correlação significativa com o IAF. Assim, qualquer um desses métodos, exceto o SDL4, pode se constituir em instrumento para estimar o ICD, a partir de fotografias digitais. A inclusão no Sidelook 1.1 do algoritmo de detecção automática do limiar, alcançado pelo mais alto contraste entre os pixels que representam o céu e o dossel, utilizando pixels, localizados nas bordas das fotografias (Nobis \& Hunziker, 2005), pode ter contribuído para melhor classificação das fotografias com o uso desse aplicativo.

Os ICD estimados pelo aplicativo Erdas Imagine 8.5 (classificação não-supervisionada) e pelo GLA 2.0 não apresentaram correlação significativa com o IAF nos dados obtidos aos 81 meses. O Erdas é um aplicativo desenvolvido para análise de imagens de satélite e fotografias aéreas, e foi recentemente utilizado na análise de fotografias digitais obtidas com câmeras fotográficas digitais comuns (Chaves, 2005; Przeszlowska et al., 2006). Chaves (2005) constatou que, com o uso desse aplicativo, houve dificuldade em separar os pixels que representavam o céu e o dossel em povoamentos submetidos a desbaste. Imediatamente após o desbaste, o método foi eficiente em identificar a redução na cobertura de dossel, porém, não foi suficiente para detectar pequenas variações no aumento subseqüente dessa cobertura. Esse autor conseguiu identificar com maior precisão as variações na dinâmica do dossel do povoamento após o desbaste, utilizando dados de IAF, obtidos com LAI-2000, ou por meio da estimativa da transmitância da radiação, com uso de sensores lineares quânticos. $\mathrm{Na}$ análise de fotografias de pastagens com gramíneas de porte baixo, tomadas a um metro de altura do chão, Przeszlowska et al. (2006) ressaltaram a dificuldade de separar, com esse aplicativo, a vegetação verde da vegetação seca e do solo.

Tabela 1. Coeficiente de correlação linear entre o índice de área foliar (IAF), estimado com LAI-2000, e índices de cobertura de dossel (ICD), estimados por vários métodos de classificação de fotografias digitais, usando a câmera digital Sony MVC FD88 (640x480 pixels), aos 81 meses de idade, e câmera digital Sony W7 (1.280x960 pixels), aos 93 meses de idade, em povoamento de clone de E. grandis submetido a desrama e desbaste ${ }^{(1)}$.

\begin{tabular}{|c|c|c|c|c|c|c|c|c|}
\hline \multirow[t]{2}{*}{ Variáveis } & \multirow[t]{2}{*}{ IAF } & \multicolumn{7}{|c|}{ Índice de cobertura de dossel } \\
\hline & & ERDAS & GLA & SDL1 & SDL2 & SDL3 & SDL4 & SDL5 \\
\hline & & & & 81 mese & & & & \\
\hline IAF & 1,00 & $0,31^{\mathrm{ns}}$ & $0,14^{\mathrm{ns}}$ & $0,41^{*}$ & $0,42 *$ & $0,35^{*}$ & $0,24^{\mathrm{ns}}$ & $0,36^{*}$ \\
\hline ERDAS & & 1,00 & $0,51^{*}$ & $0,45^{*}$ & $0,46^{*}$ & $0,38^{*}$ & $0,43 *$ & $0,47 *$ \\
\hline GLA & & & 1,00 & $0,83^{*}$ & $0,84^{*}$ & $0,79 *$ & $0,85^{*}$ & $0,84^{*}$ \\
\hline SDL1 & & & & 1,00 & $0,99 *$ & $0,93 *$ & $0,88^{*}$ & $0,98^{*}$ \\
\hline SDL2 & & & & & 1,00 & $0,95^{*}$ & $0,84 *$ & $0,91 *$ \\
\hline SDL3 & & & & & & 1,00 & $0,89 *$ & $0,97 *$ \\
\hline SDL4 & & & & & & & 1,00 & $0,87 *$ \\
\hline SDL5 & & & & & & & & 1,00 \\
\hline & & & & 93 mese & & & & \\
\hline IAF & 1,00 & $0,52 *$ & $0,54 *$ & $0,63^{*}$ & $0,66^{*}$ & $0,67 *$ & $0,63 *$ & $0,65^{*}$ \\
\hline ERDAS & & 1,00 & $0,76^{*}$ & $0,90 *$ & $0,89 *$ & $0,90 *$ & $0,88^{*}$ & $0,89 *$ \\
\hline GLA & & & 1,00 & $0,88^{*}$ & $0,90 *$ & $0,87 *$ & $0,87^{*}$ & $0,90 *$ \\
\hline SDL1 & & & & 1,00 & $0,99 *$ & $0,96^{*}$ & $0,97 *$ & $0,98 *$ \\
\hline SDL2 & & & & & 1,00 & $0,97 *$ & $0,95^{*}$ & $0,97 *$ \\
\hline SDL3 & & & & & & 1,00 & $0,98 *$ & $1,00^{*}$ \\
\hline SDL4 & & & & & & & 1,00 & $0,99 *$ \\
\hline SDL5 & & & & & & & & 1,00 \\
\hline
\end{tabular}

(1)SDL1: níveis de cinza e limiarização manual utilizando o ponto máximo do valor de borda; SDL2: níveis de cinza e limiarização automática, com o ponto máximo do valor de borda determinado pela função "next"; SDL3: banda azul e limiarização manual; SDL4: banda azul e limiarização automática (128); SDL5: banda azul e limiarização automática, com o ponto máximo do valor de borda determinado pela função "next". ns Não-significativo. *Correlação significativa a 5\% de probabilidade. 
A baixa correlação entre o IAF e o ICD obtida com a classificação das fotografias pelo GLA pode ter ocorrido em razão de esse aplicativo ter sido desenvolvido para classificação de fotografias hemisféricas (Frazer et al., 1999). Ao analisar fotografias digitais comuns com esse método, utiliza-se um círculo em que o seu diâmetro corresponde à menor dimensão da fotografia retangular, excluindo-se o restante da área da fotografia.

Aos 93 meses de idade, quando as fotografias digitais foram registradas com câmera Sony W7, com dimensões de 1.280x960 pixels, houve correlação significativa entre o IAF e os ICD obtidos por meio de todos os métodos de classificação das fotografias, até mesmo por meio do Erdas Imagine 8.5 e do GLA 2.0 (Tabela 1). Verificaram-se, porém, maiores valores de correlação quando as fotografias foram classificadas utilizando várias metodologias com o aplicativo Sidelook 1.1 (Tabela 1), o que pode ser devido à melhor definição de cor das fotografias obtidas nessa idade. Inoue et al. (2004) observaram diferenças significativas entre as dimensões da imagem para a câmera Coolpix 900, sendo a fotografia de maior dimensão (1.280x960 pixels) considerada mais adequada, quando comparada com a dimensão de 640x480 pixels, em razão de maior precisão na captação das imagens dos elementos da copa, como folhas e galhos. No entanto, em relação à câmera Coolpix 990, esses autores não observaram diferenças significativas entre as combinações de dimensões e qualidade da fotografia (básica, normal e alta) e recomendaram o uso das dimensões $640 \times 480$ pixels para essa câmera, por requerer menor espaço na memória para armazenamento das fotografias.

Nobis \& Hunziker (2005) utilizaram fotografias de $2.048 \times 1.536 \mathrm{e} 1.600 \times 1.200$ pixels para estudos do dossel de povoamentos florestais em clima temperado. Segundo esses autores, a maior dimensão da fotografia facilita a distinção dos pixels que representam o céu e o dossel. Atualmente, em razão da evolução na capacidade de armazenamento e do processamento de dados, são menores as limitações quanto à obtenção de fotografias de maior dimensão, o que favorece a tomada de fotografias em dimensões que melhor distingam o céu e o dossel.

Outras características das fotografias podem afetar a sua classificação, como a exposição - dependente da abertura do diafragma, da velocidade do obturador e da sensibilidade do filme -, que pode aumentar o contraste entre céu e dossel (Zhang et al., 2005); a limiarização, que favorece a separação dos pixels que representam o céu e o dossel (Nobis \& Hunziker, 2005), entre outras, que constituem fatores determinantes para classificação da imagem e, conseqüentemente, no sucesso da obtenção de boas correlações entre ICD e o IAF.

Em ambas as idades de avaliação, a classificação que utilizou níveis de cinza com limiar automático, por meio do aplicativo Sidelook 1.1 (SDL2), gerou ICD melhor correlacionado com o IAF pelo fato de ter ocorrido melhor separação dos pixels que representavam o céu e o dossel (Figura 1).

As equações de regressão do IAF em função dos ICD obtidos por meio de diferentes métodos de classificação de fotografias digitais, aos 81 meses, não foram significativas para os métodos Erdas, GLA e SDL4 (respectivamente: IAF $=0,32825+1,2670$ Erdas; $\mathrm{IAF}=1,05100+0,27461 \mathrm{GLA} ; \mathrm{IAF}=0,83418+$ 0,64325SDL4). Assim, estes métodos não são recomendados quando se utilizam fotografias de menor dimensão (640x480 pixels). Nas outras equações de regressão geradas com o SDL1, SDL2, SDL3 e SDL5 (respectivamente: $\mathrm{IAF}=0,82219+0,65252 \mathrm{SDL} 1$; $\mathrm{IAF}=0,75610+0,741376 \mathrm{SDL} 2 ; \mathrm{IAF}=0,71074+$ 0,78458 SDL $3 ;$ IAF $=0,80476+0,65130$ SDL5) houve significância estatística e estes métodos podem ser utilizados para obtenção do ICD e posterior estimativa do IAF. O ICD obtido pelo SDL2 foi o que melhor se correlacionou com o IAF.

Aos 93 meses, todas as regressões com IAF, em função dos ICD obtidos pelos vários métodos de classificação das fotografias, foram significativas, até mesmo os que empregaram o Erdas e o GLA, indicando que, quando se utiliza fotografia de maior dimensão, esses métodos de classificação podem ser utilizados. As equações obtidas foram: IAF $=0,69207+$ 1,4957 Erdas; $\quad$ IAF $=0,89666+1,3747 \mathrm{GLA}$; $\mathrm{IAF}=0,70494+1,7684 \mathrm{SDL} 1 ; \mathrm{IAF}=0,62270+$ 1,8774 SDL $2 ; \quad$ IAF $=0,52184+2,0152$ SDL 3 ; $\mathrm{IAF}=0,50991+2,1696 \mathrm{SDL} 4 ; \mathrm{IAF}=0,57327+$ 1,9555SDL5. No entanto, devem ser usados, preferencialmente, métodos menos morosos e subjetivos. Assim, nesse caso, os métodos de classificação utilizando o aplicativo Sidelook $1.1 \mathrm{com}$ limiarização automática, levando-se em conta o ponto máximo do valor de borda, utilizando a função "next", são os mais recomendados. 

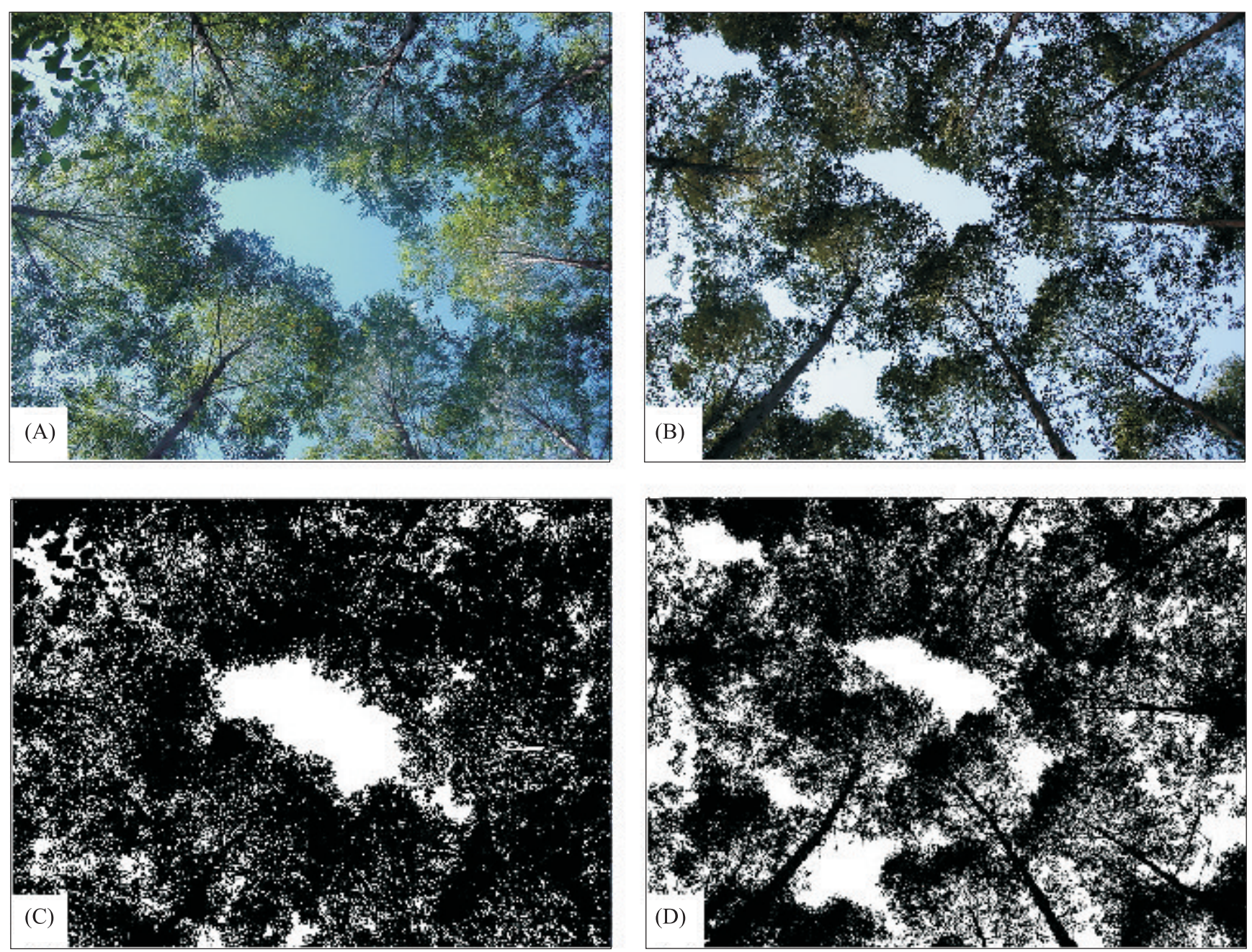

Figura 1. Fotografias obtidas com a câmera Sony MVC FD88, nas dimensões de 640x480 pixels, aos 81 meses de idade (A, C), e com a Sony W7, nas dimensões de1.280x960 pixels, aos 93 meses de idade (B, D), em seu formato original (A, B), e processadas por meio do aplicativo Sidelook 1.1, considerando níveis de cinza e determinação automática do limiar, levando-se em conta o ponto máximo do valor de borda, com uso da função "next" $(C, D)$.

\section{Conclusões}

1. O índice de cobertura de dossel obtido com fotografia digital convencional apresenta correlação significativa com o índice de área foliar.

2. O uso de fotografias digitais com maior resolução permite maior diferenciação entre céu e dossel.

3. O uso de níveis de cinza e determinação automática do limiar no Sidelook 1.1 é o método mais adequado para a classificação das fotografias digitais utilizadas.

\section{Agradecimentos}

À Capes, à Fapemig, ao CNPq e à Companhia Agrícola e Florestal Santa Bárbara Ltda., pelo apoio financeiro e logístico.

\section{Referências}

ALMEIDA, M.L. de. Desrama artificial em clones de híbridos de Eucalyptus grandis $\mathrm{x}$ E. urophylla com diferenças em arquitetura de copa. 2003. 119p. Dissertação (Mestrado) Universidade Federal de Viçosa, Viçosa. 
CHAVES, R.A. Dinâmica de copa e crescimento de plantas de clone de Eucalyptus grandis W. Hill ex Maiden submetidas a desrama artificial e a desbaste. 2005. 85p. Dissertação (Mestrado) - Universidade Federal de Viçosa, Viçosa.

CHERRY, M.; HINGSTON, A.; BATTAGLIA, M.; BEADLE, C. Calibrating the LI-COR LAI-2000 for estimating leaf area index in eucalypt plantations. Tasforests, v.10, p.75-82, 1998.

FRAZER, G.W.; CANHAM, C.D.; LERTZMAN, K.P. Gap light analyzer (GLA), version 2.0: imaging software to extract canopy structure and gap light transmission indices from true-colour fisheye photographs, users manual and program documentation. 1999. Disponível em: <http://www.rem.sfu.ca/forestry/downloads/ gap_light_analyzer.htm>. Acesso em: 10 set. 2006.

FRAZER, G.W.; FOURNIER, R.A.; TROFYMOW, J.A.; HALL, R.J. A comparison of digital and film fisheye photography for analysis of forest canopy structure and gap light transmission. Agricultural and Forest Meteorology, v.109, p.249-263, 2001.

HARDY, J.P.; MELLOH, R.; KOENIG, G.; MARKS, D.; WINSTRAL, A.; POMEROY, J.W.; LINK, T. Solar radiation transmission through conifer canopies. Agricultural and Forest Meteorology, v.126, p.257-270, 2004.

INOUE, A.; YAMAMOTO, K.; MIZOUE, N.; KAWAHARA, Y. Effects of image quality, size and camera type on forest light environment estimates using digital hemispherical photography. Agricultural and Forest Meteorology, v.126, p.89-97, 2004.

JONCKHEERE, I.; FLECK, S.; NACKAERTS, K.; MUYS, B.; COPPIN, P.; WEISS, M.; BARET, F. Review of methods for in situ leaf area index determination. I. Theories, sensors and hemispherical photography. Agricultural and Forest Meteorology, v.121, p.1935, 2004.

LEBLANC, S.G.; CHEN, J.M. A practical scheme for correcting multiple scattering effects on optical LAI measurements. Agricultural and Forest Meteorology, v.110, p.125-139, 2001.

LEBLANC, S.G.; CHEN, J.M.; FERNANDES, R.; DEERING, D.W.; CONLEY, A. Methodology comparison for canopy structure parameters extraction from digital hemispherical photography in boreal forests. Agricultural and Forest Meteorology, v.129, p.187207, 2005.

LEICA GEOSYSTEMS. ERDAS imagine. Version 8.5. Atlanta, 1999. Disponível em: <http://gi.leica-geosystems.com>. Acesso em: 10 set. 2006.

LIMA, A.P.L. Desrama artificial em clone de Eucalyptus grandis (HILL ex MAIDEN): efeitos sobre o crescimento, a dinâmica de copa e o tempo de desrama. 2003. 195p. Tese (Doutorado) Universidade Federal de Viçosa, Viçosa.
LINHARES, C.A.; PONZONI, F.J.; SHIMABUKURO, Y.E. Relação entre volume de madeira e Índice de Área Foliar em um povoamento de Pinus spp.: estudo de caso. Revista Árvore, v.24, p.47-54, 2000.

MACFARLANE, C.; COOTE, M.; WHITE, D.A.; ADAMS, M.A. Photographic exposure affects indirect estimation of leaf area in plantations of Eucalyptus globulus Labill. Agricultural and Forest Meteorology, v.100, p.155-168, 2000.

NOBIS, M. Sidelook 1.1: imaging software for the analysis of vegetation structure with true-colors photographs. 2005. Disponível em: <http://www.appleco.ch>. Acesso em: 20 set. 2006.

NOBIS, M.; HUNZIKER, U. Automatic thresholding for hemispherical canopy-photographs based on edge detection. Agricultural and Forest Meteorology, v.128, p.243-250, 2005.

PEZZOPANE, J.E.M.; REIS. G.G. dos; REIS, M.G.F.; COSTA, J.M.N. da. Caracterização da radiação solar em fragmento da Mata Atlântica. Revista Brasileira de Agrometeorologia, v.13, p.1119, 2005.

PRZESZLOWSKA, A.; TRLICA, M.J.; WELTZ, M.A. Near-ground remote sensing of green area index on the shortgrass prairie. Rangeland Ecology and Management, v.59, p.422-430, 2006.

VILLA NOVA, N.A.; MOREIRA, P.R.; PEREIRA, A.B. Eficiência de captura de energia solar por dosséis de Eucalyptus pellita F. Muell sob várias densidades de plantio. Revista Brasileira de Agrometeorologia, v.11, p.269-274, 2003.

WAGNER, S. Calibration of grey values of hemispherical photographs for image analysis. Agricultural and Forest Meteorology, v.90, p.103-117, 1998.

WAGNER, S. Relative radiance measurements and zenith angle dependent segmentation in hemispherical photography. Agricultural and Forest Meteorology, v.107, p.103-115, 2001.

WEISS, M.; BARET, F.; SMITH, G.J.; JONCKHEERE, I.; COPPIN, P. Review of methods for in situ leaf area index (LAI) determination. II. Estimation of LAI, errors and sampling. Agricultural and Forest Meteorology, v.121, p.37-53, 2004.

XAVIER, A.C.; SOARES, J.V.; ALMEIDA, A.C. de. Variação do Índice de Área Foliar em clones de eucalipto ao longo de seu ciclo de crescimento. Revista Árvore, v.26, p.421-427, 2002.

XAVIER, A.C.; VETTORAZZI, C.A. Leaf area index of ground covers in a subtropical watershed. Scientia Agricola, v.60, p.425431, 2003.

ZHANG, Y.; CHEN, J.M.; MILLER, J.R. Determining digital hemispherical photograph exposure for leaf area index estimation. Agricultural and Forest Meteorology, v.133, p.166-181, 2005. 